\title{
Effects of tyrosine kinase inhibitor-masitinib mesylate on canine mammary tumour cell lines
}

\author{
Fulya Ustun-Alkan ${ }^{1}$, Tülay Bakırel ${ }^{1}$, Oya Üstüner ${ }^{1}$, Ceren Anlas ${ }^{1}$, \\ Suzan Cinar ${ }^{2}$, Funda Yıldırım ${ }^{3}$, Aydın Gürel ${ }^{3}$ \\ ${ }^{1}$ Department of Pharmacology and Toxicology, ${ }^{3}$ Department of Pathology, \\ Faculty of Veterinary Medicine, Istanbul University-Cerrahpasa, 34500, Istanbul, Turkey \\ ${ }^{2}$ Department of Immunology, Aziz Sancar Institute of Experimental Medicine, \\ Istanbul University, 34093, Istanbul, Turkey
}

Received: February 5, $2021 \quad$ Accepted: July 5, 2021

\begin{abstract}
Introduction: Masitinib mesylate, a selective tyrosine kinase inhibitor of the c-KIT receptor, is used for the treatment of mast cell tumours in dogs. Masitinib has previously been investigated in various cancers; however, its potential anticancer effect in canine mammary tumours (CMTs) is unknown. In the present paper, we investigated the antiproliferative effect of masitinib in CMT cells and its possible mechanisms of action. Material and Methods: The effect of masitinib on the proliferation of CMT-U27 and CMT-U309 cells was assessed by MTT assay and DNA fragmentation. Flow cytometric analysis was used to measure the effect of masitinib on apoptosis and the cell cycle. Additionally, vascular endothelial growth factor levels (VEGF) were measured, and the proliferation marker Ki-67 was visualised in immunocytochemical stainings in CMT cells. Results: Treatment with masitinib inhibited the proliferation of CMT cells in a concentration-dependent manner. Maximal apoptotic activity and DNA fragmentation were observed at approximately $\mathrm{IC}_{50}$ of masitinib in both cell lines. In addition, cell cycle distribution was altered and VEGF levels and Ki-67 proliferation indices were decreased in masitinib-treated cells in comparison with control cells. Conclusion: In this study, masitinib suppressed cell proliferation concomitantly via induction of apoptosis and cell cycle arrest by decreasing VEGF levels and the Ki-67 proliferation index in CMT-U27 and CMT-U309 cells in vitro, suggesting its potential as a therapeutic tool in the clinical setting of mammary cancer treatment in dogs.
\end{abstract}

Keywords: tyrosine kinase inhibitor, masitinib, canine mammary cancer, apoptosis, VEGF.

\section{Introduction}

The remarkable progress in understanding the molecular aspects of cancer has led to the development of novel therapies, which refer to new therapeutic agents targeting specific pathways in cancer cells $(16,28)$. Tyrosine kinases are the key mediators of the normal and cancer cell signalling network and they have been shown to be involved in the regulation of cellular proliferation, differentiation and angiogenesis. In addition, they also play an important role in the development, progression and metastasis of several types of cancer (2). Based on the role of receptor tyrosine kinases (RTKs) in the regulation of various cellular processes, especially dysregulation of RTK signalling in cancer, RTKs are considered to be a relevant target for anticancer drug research and development (15). Several oncogenic tyrosine kinases such as vascular endothelial growth factor (VEGF), epidermal growth factor receptor (EGFR), platelet-derived growth factor receptor (PDGFR), c-KIT and c-Met have been found to be dysregulated in canine mammary tumours $(11,12)$. Although dysregulation of RTKs in dogs is not well known, similar mechanisms of RTK dysregulation were found in canine tumours when compared with their human counterparts. Clinically, canine mammary tumours (CMTs) and human breast tumours share many similar biological behaviours and the evidence of canine genome similarity to the human genome has emphasised the $\operatorname{dog}$ as an attractive alternative model for testing cancer therapeutic agents $(9,20)$. Based on the above-mentioned findings, it has been suggested that CMTs may be potential targets for tyrosine kinase inhibitor (TKI) therapy.

Mammary tumours are the most common neoplasm in female dogs and represent a serious problem in veterinary medicine (9). Development of mammary 
tumours depends on many factors such as alterations in the expression of hormone receptors, dysregulation of cellular differentiation or insufficient apoptosis (3). Despite recent advances multimodally combining surgery, supportive, radio-, and systemic chemotherapy in mammary cancer, the overall prognosis is still poor, the disease-free time short and survival rate low (9). Therefore, novel and innovative approaches are needed for the treatment of canine mammary cancer.

In the two decades since TKIs were discovered they have taken up a role in cancer treatment, and their therapeutic importance is increasing. These inhibitors are small molecules targeting a specific and/or broad spectrum of RTKs that block crucial intracellular signalling pathways in tumour cells, leading to deregulation of key cell functions (15). They show a much higher selectivity towards tumour cells than normal cells when compared with traditional chemotherapy. TKIs provide a wide therapeutic window with low toxicity to healthy tissues and improve the patient's quality of life (2). Many in vitro and in vivo studies have been conducted to evaluate the clinical efficacy and effectiveness of TKIs against different tumours. Most of them showed good antitumour efficacy against various cancers (17, 23, 28, 29). Therefore, a great number of studies are focused on expanding the use of TKIs to other cancers.

Masitinib mesylate, formerly known as AB1010, is the first potent and selective TKI. It was approved by the European Medicines Agency in veterinary medicine for treatment of canine mast cell tumours about 15 years ago and targets mainly c-Kit receptor (c-KitR) and, to a lesser extent, other tyrosine kinase receptors such as lymphocytespecific kinase (Lck), Lck/Yes-related protein (LYn), platelet-derived growth factor receptor-alpha/beta $(\mathrm{PDGFR} \alpha / \beta)$, and fibroblast growth factor receptor 3 (FGFR3) (16). It is involved in controlling the proliferation, differentiation and degranulation of mast cells and therefore indirectly their released mediators, via direct antiproliferative and pro-apoptotic actions, which are involved in various mechanisms that facilitate tumour progression (26). As a corollary to its favourable results against canine mast cell tumours, masitinib is also considered to have potential against several human malignancies and is being researched accordingly (5, 16). Additionally, it has previously been shown to have antiproliferative activity and induce apoptosis in a variety of animal tumour cell lines $(6,14,15)$. However, most canine malignant mammary tumours express a significant level of c-KIT proto-oncogene, which is the main target of masitinib (13). Studies have shown that masitinib exhibited anticancer activity by inhibiting RTK targets and by disturbing RTK signalling pathways associated with cancer progression and metastasis (6). Considering these findings in canine tumours, we hypothesised that masitinib could be effective in the treatment of canine mammary tumours. Therefore, the purpose of this study was to investigate the effects of masitinib on CMT cell lines and its possible mechanism of action.

\section{Material and Methods}

Cell culture and chemicals. Masitinib mesylate was kindly provided by AB Science (Paris, France). A stock solution $(20 \mathrm{mM})$ was made in $100 \%$ dimethyl sulphoxide (DMSO) and stored at $-20^{\circ} \mathrm{C}$, then serially diluted in Dulbecco's modified Eagle's medium-F12 (DMEM-F12) immediately prior to the experiments. In the cell culture medium, the maximum concentration of DMSO did not exceed $0.2 \%$ as this concentration had no effect on cell proliferation, and this concentration was used as control. The canine mammary carcinoma cell line CMT-U27 and canine mammary spindle-cell tumour cell line CMT-U309 were kindly donated by Prof. Dr. Eva Hellmén (Uppsala University, Sweden). Cell lines were maintained in DMEM-F12 supplemented with $1 \%(\mathrm{v} / \mathrm{v})$ L-glutamine, $10 \%(\mathrm{v} / \mathrm{v})$ foetal bovine serum (FBS) and $100 \mathrm{U} / \mathrm{mL}$ penicillin and streptomycin $100 \mu \mathrm{g} / \mathrm{mL}$ in a humidified atmosphere containing $5 \% \mathrm{CO}_{2}$ at $37^{\circ} \mathrm{C}$. Adherent cells were harvested by trypsin/EDTA solution when reaching approximately 80-90\% confluence. All reagents were purchased from Gibco (Invitrogen, Carlsbad, CA, USA) unless otherwise indicated.

Cell proliferation test. Cell viability was determined using a trypan blue solution with the Cedex XS cell counter system (Innovatis, Roche, Mannheim, Germany) before seeding the cells. Cell viability was measured using the MTT (3-(4,5-dimethylthiazol-2-yl)2,5-diphenyltrazolium bromide) assay which is based on the activity of mitochondrial dehydrogenases. Canine mammary tumour CMT-U27 and CMT-U309 cells were seeded at $1 \times 10^{4}$ cells/well in medium and incubated at $37^{\circ} \mathrm{C}$ in a humidified atmosphere of $5 \% \mathrm{CO}_{2}$ for $24 \mathrm{~h}$ and then treated with varying concentrations $(0.25-32 \mu \mathrm{M})$ of masitinib for 24, 48 and $72 \mathrm{~h}$. At the end of the treatments, $10 \mu \mathrm{L}$ of MTT solution from the MTT Cell Proliferation Kit (Roche Applied Science, Mannheim, Germany) was added to each well. After $4 \mathrm{~h}$ of incubation, the MTT formazan crystals were solubilised with $10 \% \mathrm{SDS}$ in $0.01 \mathrm{M} \mathrm{HCl}$ solution. Following overnight incubation, the absorbance of the wells was measured by a FilterMax F5 microplate reader (Molecular Devices, San Jose, CA, USA) at $595 \mathrm{~nm}$. All samples were tested in triplicate, and the mean for each experiment was calculated. The effect of masitinib on growth inhibition was calculated as cell viability (\%) relative to control cells. The mean value of triplicate experiments for each dose was used to calculate the concentration of drug needed for inhibition of cell viability $\left(\mathrm{IC}_{20}, \mathrm{IC}_{50}\right.$ and $\left.\mathrm{IC}_{80}\right)$ as determined by CalcuSyn software (Biosoft, Cambridge, UK).

DNA fragmentation assay. Cellular DNA fragmentation was assessed by analysis of the cytoplasmic histone-associated Cell Death Detection ELISA Plus Kit (Roche Applied Science, Mannheim, Germany) according to the manufacturer's instructions. The relative amounts of mono- and oligonucleosomes in the cytoplasmic fraction of cell lysates which were 
generated from the apoptotic cells were quantified using mouse monoclonal antibodies directed against DNA and histones. The experimental procedure was repeated as noted in the proliferation assay. At the end of the $72 \mathrm{~h}$ treatment period, the absorbance of the wells was measured at $405 \mathrm{~nm}$ with a reference wavelength at $490 \mathrm{~nm}$ by the FilterMax F5 microplate reader. Samples were tested in triplicate and the fold change in apoptosis was calculated as optical density (OD) masitinib/OD control.

Annexin $\mathrm{V}$ apoptosis assay. Flow cytometric analysis of programmed cell death was performed using an annexin V-fluorescein isothiocyanate (FITC) Apoptosis Detection Kit (Becton Dickinson, Franklin Lakes, NJ, USA) and Calibur fluorescence-activated cell sorting apparatus (Becton Dickinson). Briefly, $1 \times 10^{5} / \mathrm{mL}$ cells for each line were seeded into 24 -well plates (Jet Biofil, Guangzhou, China) and incubated overnight. The medium was replaced with varying concentrations $(0.25-8 \mu \mathrm{M})$ of masitinib for $72 \mathrm{~h}$. The cells were then trypsinised and washed twice each with PBS and subsequently stained with annexin V-FITC and propidium iodide (PI) according to the manufacturer's instructions. The samples were analysed by FACScan flow cytometer (BD Bioscience, Franklin Lakes, NJ, USA). Data were processed and displayed using the CellQuest WinMDI software (BD Bioscience).

Cell cycle analysis. Cell cycle distribution was detected using a CycleTest Plus DNA Reagent kit (Becton Dickinson) according to the manufacturer's instructions. The experimental procedure was the same as described in the apoptosis assay. At the end of the $72 \mathrm{~h}$ incubation period, cells were collected by trypsinisation and washed with buffer solution, then centrifuged at $400 \times \mathrm{g}$. The cell pellets were incubated and subsequently stained according to the manufacturer's protocol. Analyses were performed using the FACScan flow cytometer at an excitation wavelength of $488 \mathrm{~nm}$, while the data were analysed with ModFit LT cell cycle analysis software (BD Bioscience).

Ki-67 immunocytostaining. In order to measure the effect of masitinib on the proliferation index of CMT cells, $\mathrm{Ki}-67$, which is an excellent proliferation marker, was used to determine the growth fraction of cell populations. Briefly, cells were cultured on sterile glass coverslips and incubated overnight. Then, the medium was replaced with fresh medium and masitinib at 2, 4 and $8 \mu \mathrm{M}$ was added and the solution was incubated for $72 \mathrm{~h}$. At the end of the treatment, cells were fixed with methanol and the endogenous peroxidase activity was inactivated by $0.3 \% \mathrm{H}_{2} \mathrm{O}_{2}$. Afterwards, immunocytochemical staining was performed using Ki-67 human monoclonal antibody (cat. no 14-5699-82, clone 20Raj1, eBioscience, San Diego, CA, USA). After washing in PBS, the cover slips were incubated with a Histostain Plus IHC secondary antibody kit (cat. no 85-8943, Invitrogen, Camarillo, CA. USA) for $20 \mathrm{~min}$ at room temperature. The primary antibody was omitted for negative controls. Finally, cells were incubated with $\mathrm{DAB}$ complexes according to the manufacturer's protocol and then counterstained with
Mayer's haematoxylin and mounted onto slides. Intensity of Ki-67 antigen expression was evaluated under a BX50 light microscope (Olympus, Tokyo, Japan). Positive cells were identified by distinct brown nuclear staining. The proliferation index (PI) was defined as the number of Ki-67 positive cells divided by the total number of tumour cells $\times 100 \%$. At least 1,000 CMT cells per slide were counted at $40 \times$ magnification.

Vascular endothelial growth factor measurement. To assess the potential impact of masitinib treatment on angiogenesis, VEGF levels in conditioned media were measured. The VEGF concentrations were evaluated using a Quantikine Canine VEGF Immunoassay canine ELISA kit (R\&D Systems, Minneapolis, MN, USA). This kit includes recombinant canine VEGF and anti-canine VEGF antibodies. The sensitivity of the VEGF assay was $19.5 \mathrm{pg} / \mathrm{mL}$, and the inter- and intra-assay coefficients of variation were $3.01-9.10 \%$ and $4.80-10.49 \%$, respectively $(n=7)$. In brief, cells were seeded in 24 -well plates at a density of $1.0 \times 10^{5}$ cells/well. Cells were incubated for $24 \mathrm{~h}$, after which the medium was replaced with medium containing varying concentrations $(0.25-8 \mu \mathrm{M})$ of masitinib. The plates were then incubated for $72 \mathrm{~h}$, after which the supernatants were collected and stored at $-80^{\circ} \mathrm{C}$ until analysis. The VEGF immunoassay was performed in accordance with the manufacturer's instructions. The samples and VEGF standards were assayed in duplicate. The OD of the wells was measured at $450 \mathrm{~nm}$ by the FilterMax microplate reader. A calibration curve was generated by plotting the mean OD of each VEGF standard against concentrations, and the VEGF concentrations $(\mathrm{pg} / \mathrm{mL})$ in the sample supernatants were determined according to the curve. The VEGF concentration was not normalised to cell numbers at the end of the incubation period.

Statistical analysis. Data were analysed using one-way analysis of variance (ANOVA), and the statistical significances between groups were analysed by Tukey's multiple comparison test in SPSS 15.0 (SPSS Inc., Chicago, IL, USA) and the results were expressed as the mean $\pm \mathrm{SE}$. Values for $\mathrm{P}$ of less than 0.05 were considered to be statistically significant.

\section{Results}

To determine the effects of masitinib on cell viability of CMT cells, the MTT assay was used. The results showed that masitinib reduced the cell count at the end of the 24, 48 and $72 \mathrm{~h}$ treatments (Fig. 1). Significant decreases in cell viability were found at $4 \mu \mathrm{M}$ concentrations of masitinib in both cell lines compared to the control group at $24 \mathrm{~h}$, and at 48 and $72 \mathrm{~h}$ significant declines in cell viability were found at concentrations $\geq 8 \mu \mathrm{M}(\mathrm{P}<0.001)$ when compared to the control group at the same time points. Masitinib induced a dose-dependent cytotoxic effect in both CMT cell lines with average $\mathrm{IC}_{50}$ values of $7.498 \pm 0.478$ and $8.545 \pm 0.368$ at $72 \mathrm{~h}$ in CMT-U27 and CMT-U309 cells, respectively. 
The $\mathrm{IC}_{50}$ was found to decrease with increasing exposure time to masitinib for both cell lines. Inhibitory concentration values $\left(\mathrm{IC}_{20}, \mathrm{IC}_{50}\right.$ and $\left.\mathrm{IC}_{80}\right)$ of masitinib were calculated using the CalcuSyn application (Biosoft, Cambridge, UK) from the dose response curves for the cell lines after 24,48 and $72 \mathrm{~h}$ (Table 1).

Table 1. $\mathrm{IC}_{20}, \mathrm{IC}_{50}$ and $\mathrm{IC}_{80}$ values of masitinib in CMT-U27 and CMT-U309 cells as measured by the MTT assay

\begin{tabular}{cccccc}
\hline Cell line & Treatment & Time & $\mathrm{IC}_{20}(\mu \mathrm{M})$ & $\mathrm{IC}_{50}(\mu \mathrm{M})$ & $\mathrm{IC}_{80}(\mu \mathrm{M})$ \\
\hline \multirow{3}{*}{ CMT-U27 } & \multirow{2}{*}{ Masitinib } & $48 \mathrm{~h}$ & 2.00 & 9.129 & 41.668 \\
& & $72 \mathrm{~h}$ & 2.868 & 7.498 & 19.60 \\
\hline \multirow{3}{*}{ CMT-U309 } & \multirow{2}{*}{ Masitinib } & $48 \mathrm{~h}$ & 3.097 & 8.871 & 25.405 \\
& & $72 \mathrm{~h}$ & 2.471 & 8.545 & 29.544 \\
\hline
\end{tabular}

To determine whether apoptosis played a role in the masitinib-mediated weakening of CMT cell proliferation, the appearance of DNA fragmentation after $72 \mathrm{~h}$ incubation was quantitatively analysed. Masitinib at around $\mathrm{IC}_{50}(8 \mu \mathrm{M})$ in both cell lines significantly increased the fragmentation of histone-associated DNA in CMT cells and this was accompanied by a significant increase in OD values from $0.63 \pm 0.07$ to $2.69 \pm 0.09$ in CMT-U27 cells and from $0.97 \pm 0.09$ to $2.68 \pm 0.16$ in CMT-U309 cells (Fig. 2).

To measure and characterise cell death, a quantitative apoptotic cell death assay was performed via doublestained flow cytometry. Consistently with the MTT assay, flow cytometric analyses showed a dose-dependent increase in the percentage of apoptotic cells (the sum of early and late apoptotic cells) in masitinib-treated cells. Masitinib-treated CMT-U27 cells had a higher apoptosis rate of $66.70 \%$ compared to the $16.10 \%$ rate in CMT-U309 cells and the percentage of early apoptotic cells significantly increased at $8 \mu \mathrm{M}$ and at 4 and $8 \mu \mathrm{M}$ in masitinib-treated CMT-U27 and CMT-U309 cells, respectively (Fig. 3).

To examine additional mechanisms by which masitinib inhibits cell growth, the cell-cycle distribution in CMT cells was evaluated after drug treatment. At $8 \mu \mathrm{M}$ concentration, which was approximately $\mathrm{IC}_{50}$ of masitinib in both cell lines, masitinib counteracted cellcycle progression in CMT cells and increased the proportion of $\mathrm{G}_{0} / \mathrm{G}_{1}$ cells to $78 \%$ and $86 \%$ ( $v \mathrm{~s}$. the control) in CMT-U27 and CMT-U309 cell lines, respectively (Fig. 4). A marked increase in the $\mathrm{G}_{0} / \mathrm{G}_{1}$ population and a decrease in the $S$ phase population after treatment illustrates that masitinib blocked the $G_{1}-S$ transition.

To assess tumour cell proliferation in correlation with the cell cycle, cells with Ki-67-positive nuclear immunostaining were counted. Immunocytochemical staining revealed that $\mathrm{Ki}-67$ protein expression was suppressed with increasing concentrations of masitinib (Fig. 5A-F). A lower Ki-67 index was calculated after treatment with masitinib in comparison with the control (Fig. 6).

Table 2. VEGF concentrations in the supernatant of control and masitinib treated CMT cells after $72 \mathrm{~h}$

\begin{tabular}{lll}
\hline $\begin{array}{l}\text { Masitinib } \\
\text { concentration }\end{array}$ & $\begin{array}{l}\text { CMT-U27 VEGF } \\
\text { concentration }(\mathrm{pg} / \mathrm{mL})\end{array}$ & $\begin{array}{l}\text { CMT-U309 VEGF } \\
\text { concentration }(\mathrm{pg} / \mathrm{mL})\end{array}$ \\
\hline Control & $536.5 \pm 19.01$ & $287.8 \pm 14.25$ \\
$0.25 \mu \mathrm{M}$ & $504.64 \pm 21.92$ & $247.38 \pm 14.98$ \\
$0.5 \mu \mathrm{M}$ & $439.48 \pm 13.66^{*}$ & $221.79 \pm 10.32^{* *}$ \\
$1 \mu \mathrm{M}$ & $440.28 \pm 23.13^{*}$ & $167.59 \pm 8.05^{* * *}$ \\
$2 \mu \mathrm{M}$ & $405.72 \pm 21.05^{* *}$ & $154.90 \pm 13.63^{* * *}$ \\
$4 \mu \mathrm{M}$ & $399.87 \pm 6.94^{* * *}$ & $99.59 \pm 16.38^{* * *}$ \\
$8 \mu \mathrm{M}$ & $102.74 \pm 10.62^{* * *}$ & $67.97 \pm 3.86^{* * *}$ \\
\hline${ }_{-} \mathrm{P}<0.05 ;{ }^{* *}-\mathrm{P}<0.01 ; * * *$ \\
\hline
\end{tabular}

To determine the effect of masitinib on angiogenesis, the concentration of VEGF was measured in control and masitinib-treated cells. The control cells expressed high levels of VEGF over the $72 \mathrm{~h}$ period in both cell lines. The VEGF levels in control cells for CMT-U27 and CMT-U309 were $536.5 \mathrm{pg} / \mathrm{mL}$ and $287.8 \mathrm{pg} / \mathrm{mL}$, respectively. However, the measured cell culture supernatant VEGF levels fell with rising concentrations of masitinib (Table 2) and statistical significance was observed at much lower $\mathrm{IC}_{50}$ masitinib concentrations in both cell lines.
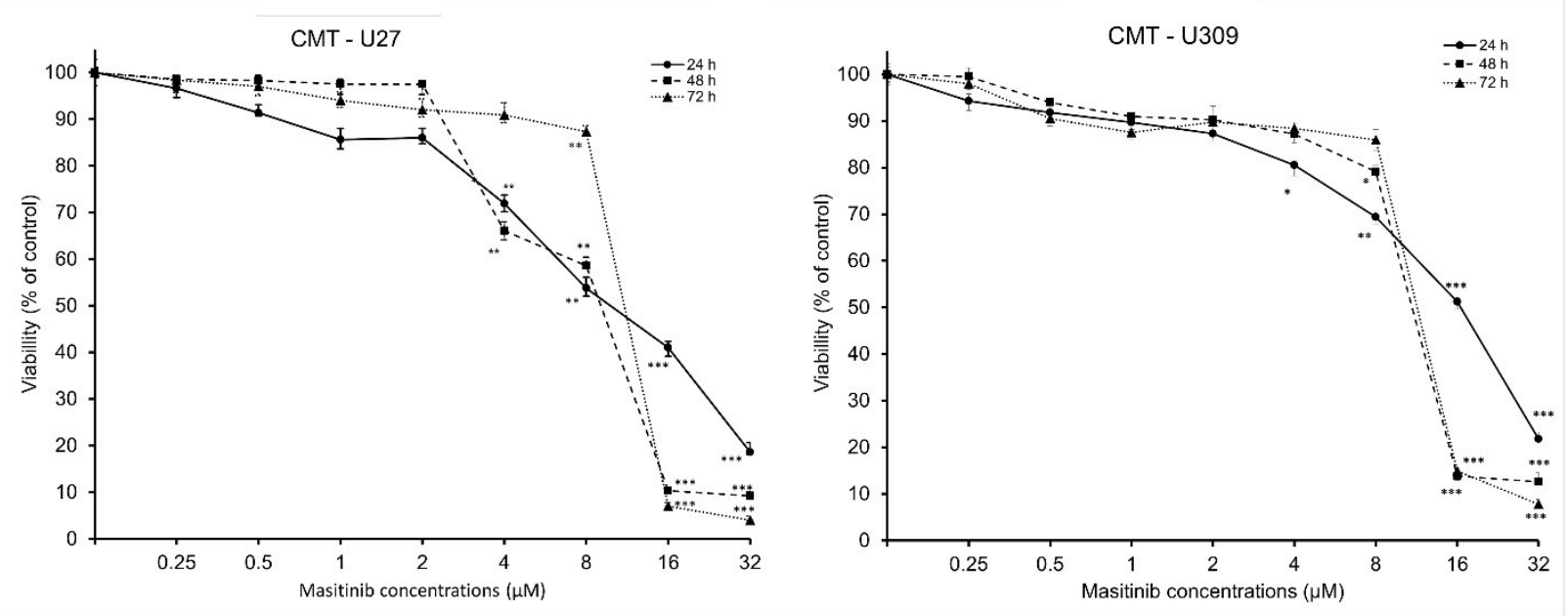

Fig. 1. Effects of masitinib treatment on cell viability of CMT-U309 and CMT-U27 cells. Data are expressed as mean percentage of cell viabilities \pm standard error (SE) from three individual experiments. * $-\mathrm{P}<0.05 ; * *-\mathrm{P}<0.01 ; * * *-\mathrm{P}<0.001$ compared to control 


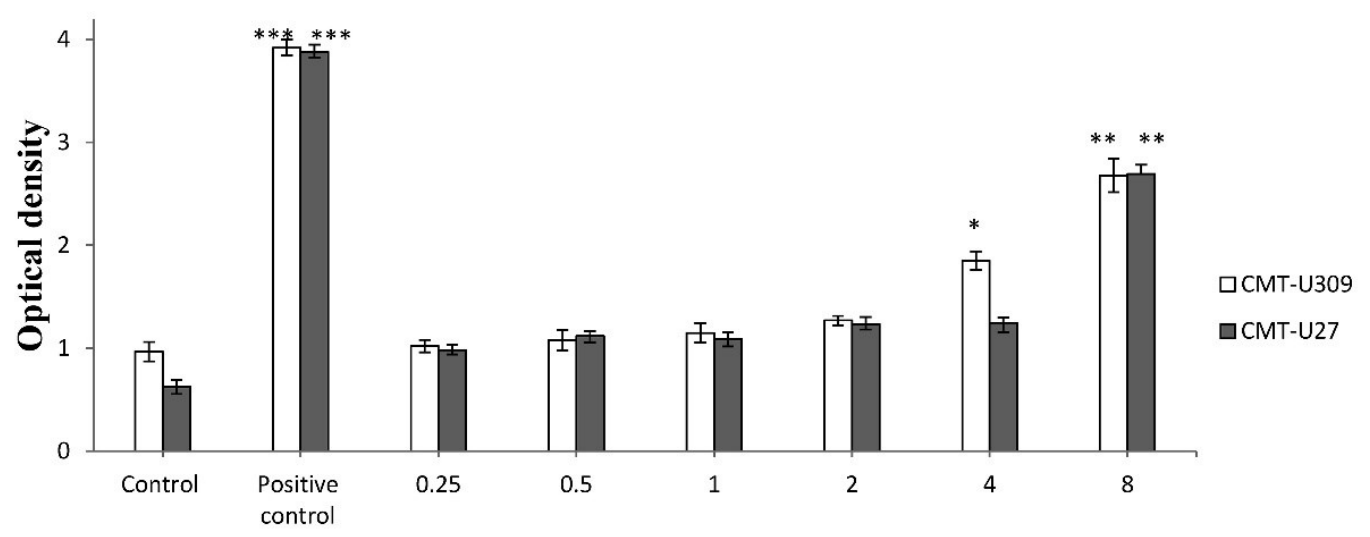

Masitinib $(\mu \mathrm{M})$

Fig. 2. Effects of masitinib on DNA fragmentation of CMT-U27 and CMT-U309 cells. Data are expressed as mean OD values \pm standard error from three individual experiments. ${ }^{*}-\mathrm{P}<0.05 ; * *-\mathrm{P}<0.01 ; * * *-\mathrm{P}<0.001$ compared to control
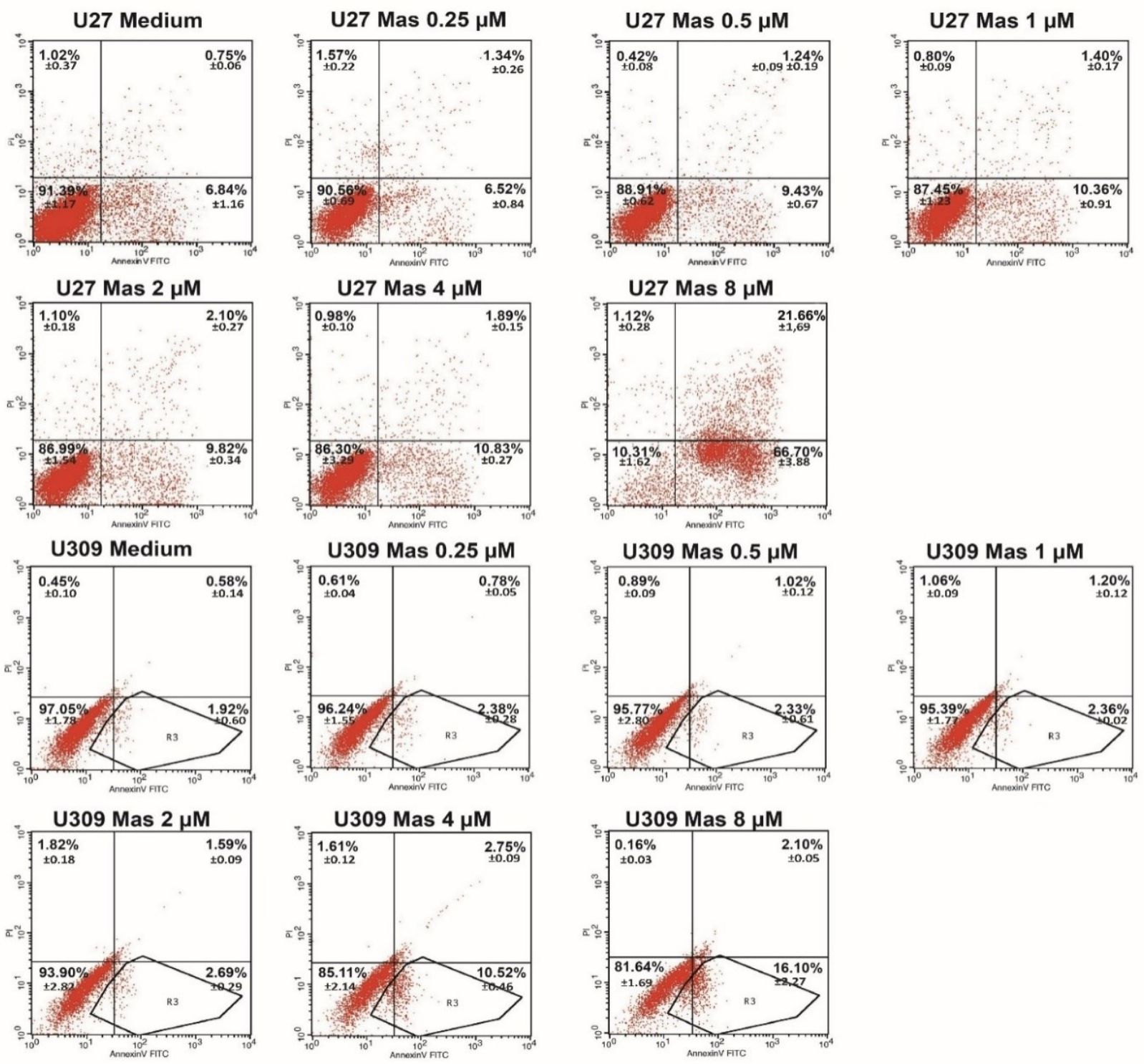

Fig. 3. Flow cytometric analysis after incubation with masitinib $(0-8 \mu \mathrm{M})$ for $72 \mathrm{~h}$ as representative profiles of annexin-V-FITC/PI staining of CMT-U27 cells and CMT-U309 cells. The lower left quadrant of the histogram shows viable cells (unstained by either fluorochrome) and the lower right one represents early apoptotic (annexin-V-positive) cells, indicating the translocation of phosphatidylserine to the external cell surface. The upper right quadrant represents late apoptotic (annexin-V-and PI-positive) cells, and the upper left one shows necrotic (PI-positive) cells. The numbers represent the mean percentage of cells $(\%) \pm$ standard error 


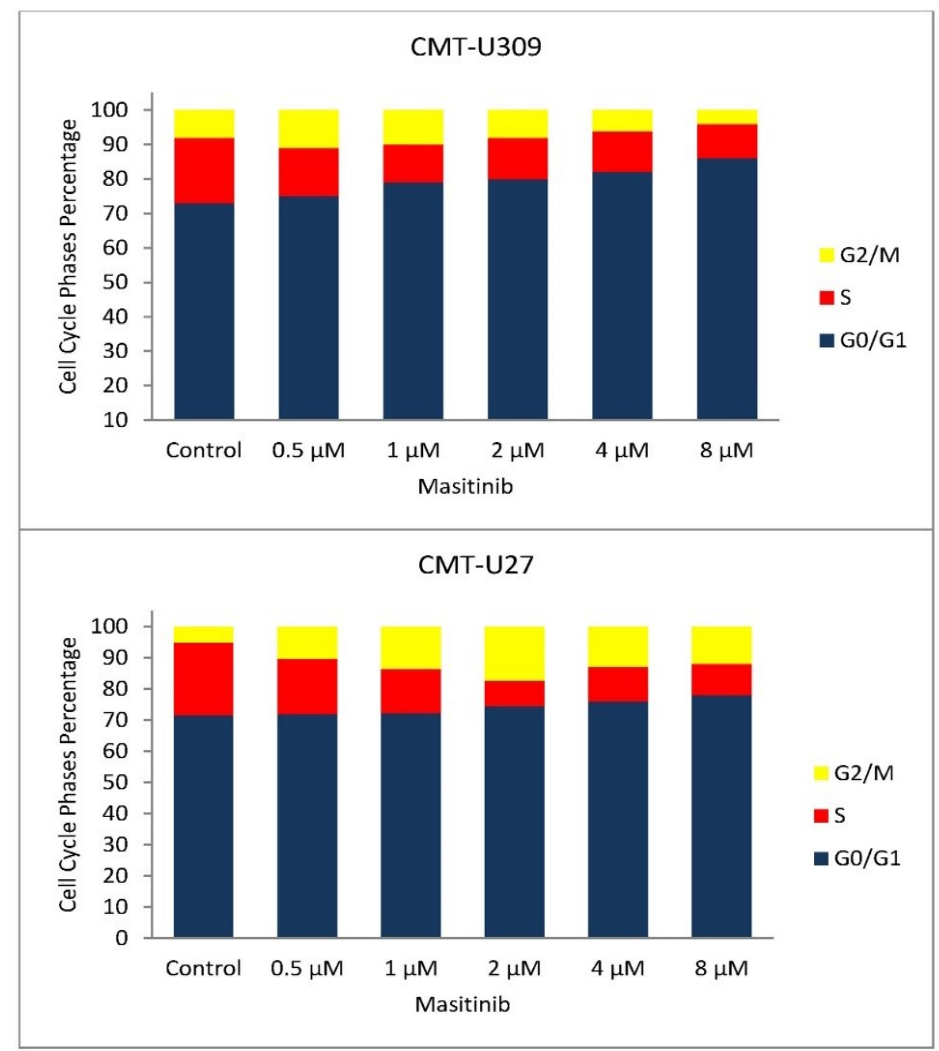

Fig. 4. Effects of masitinib treatment on the percentage of the total cell population in each phase of the cell cycle of CMT-U27 and CMT-U309 cells
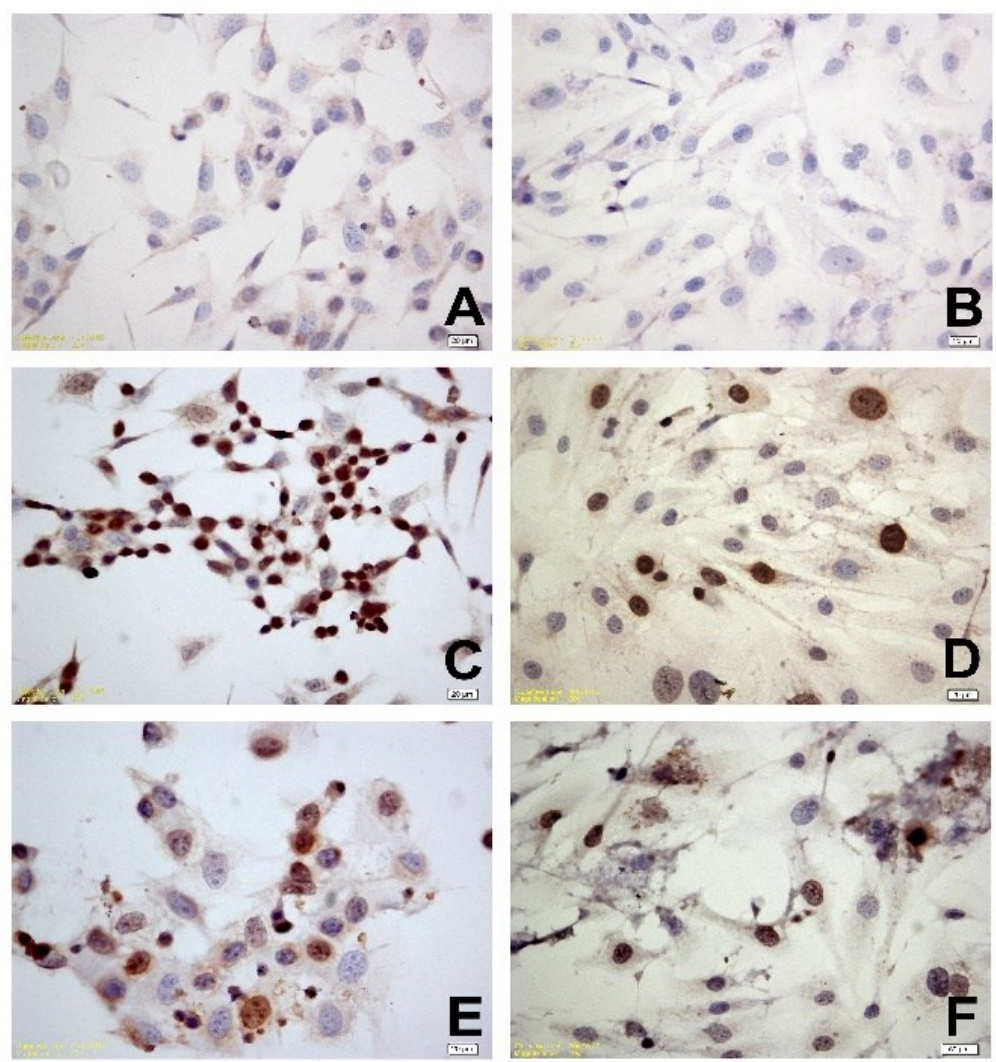

Fig. 5. Immunocytochemical staining of Ki-67 in CMT-U27 and CMT-U309 cell lines. Bar $=20 \mu \mathrm{m}$. A - negative control, CMT-U27; B - negative control, CMT-U309; $\mathrm{C}$ - control high immunopositivity of Ki-67 in CMT-U27; D - control, high immunopositivity of Ki-67 in CMT-U309; E - IC 50 masitinib, low immunopositivity of Ki-67 in CMT-U27; $\mathrm{F}-\mathrm{IC}_{50}$ masitinib, low immunopositivity of Ki-67 in CMT-U309 


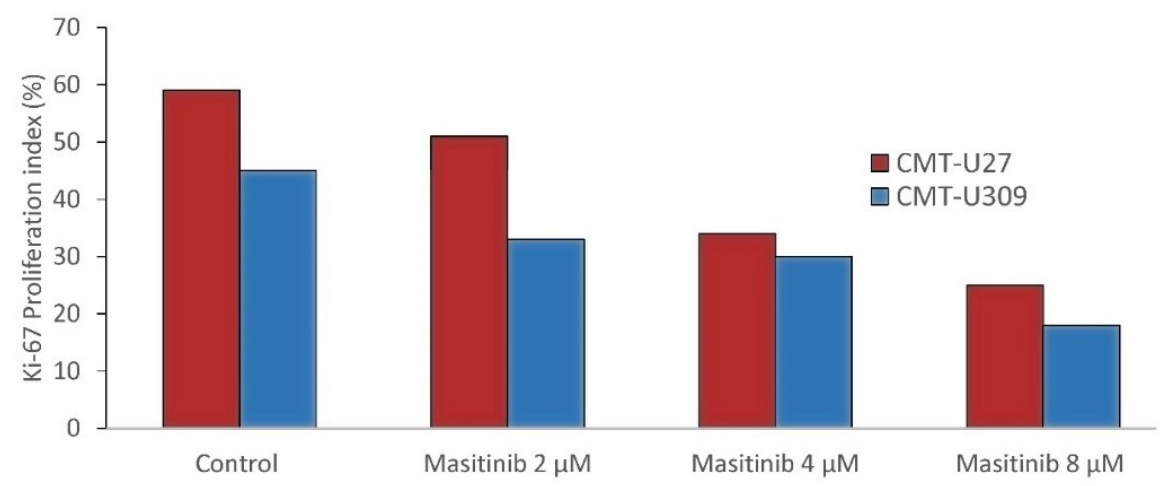

Fig. 6. Effects of masitinib treatment on the proliferation index of CMT-U27 and CMT-U309 cells measured from Ki-67-stained slides

\section{Discussion}

Masitinib mesylate is a potent, orally bioavailable, and selective inhibitor of c-Kit receptor. It has demonstrated good clinical efficacy in unresectable mast cell tumours in dogs and is under clinical assessment as a therapeutic agent in several human cancers which involve c-Kit proto-oncogene mutations similar to those in canine neoplasms $(16,21)$. The promising clinical efficiency of masitinib in c-Kit-positive mast cell tumours has encouraged preclinical studies and clinical evaluations of masitinib in many other canine malignancies $(6,8,18)$. The c-Kit proto-oncogene is reported to be frequently expressed in canine malignant mammary tumours (13). We have investigated the therapeutic potential of masitinib in canine mammary cancer prompted by these reports. In the present study, we analysed the effects of masitinib on cell proliferation, apoptosis, cell cycle and VEGF secretion in CMT cell lines. Masitinib induced a dose-dependent inhibition of cell growth in both CMT cell lines, and these results suggest that CMT cells are sensitive to masitinib and that it is an effective agent for suppressing the growth of CMT cells in vitro. In the present study, the calculated $\mathrm{IC}_{50}$ values of masitinib are similar to the $\mathrm{nM}$ to low $\mu \mathrm{M}$-ranging $\mathrm{IC}_{50}$ values of masitinib found in various cancer cell lines treated with the preparation $(6,15,18)$. The reported $\mathrm{IC}_{50}$ values of masitinib for canine oral fibrosarcoma cell lines were 9.1 and $12.0 \mu \mathrm{M}(18)$ and for canine haemangiosarcoma cell lines were $8.56,9.41$ and $10.65 \mu \mathrm{M}(15)$. In another in vitro study, in which masitinib was tested in several lines, the reported $\mathrm{IC}_{50}$ values ranged from $0.03 \mu \mathrm{M}$ to $16.8 \mu \mathrm{M}$ (26). In many instances, the exposure concentrations used in vitro have little relevance to the maximum plasma concentrations achieved in vivo. Pharmacokinetic studies in healthy beagles have shown that masitinib at an oral dose of $10 \mathrm{mg} / \mathrm{kg}$ had a half-life of $3.2 \mathrm{~h}$ with a peak plasma concentration of approximately 1.3-1.5 $\mu \mathrm{M}$ (7). Although the reported plasma concentration is lower than the $\mathrm{IC}_{50}$ value obtained in our experiments, clinical studies would be required to determine whether masitinib could be effective against canine mammary tumours because in vivo, important factors such as absorption, distribution, protein binding, metabolic activation and immune response could contribute to treatment efficacy or toxicity. However, in the present study it has been demonstrated that CMT cells produce VEGF, which is correlated with higher vascular permeability that could lead to a preferential accumulation of masitinib, so it is possible that the concentrations in the tumour cells can be higher than in plasma.

To explore the mechanism of masitinib-induced growth inhibition in CMT cells, we examined the effects of masitinib on apoptosis in CMT cells. Apoptotic activity was shown to increase rapidly close to $\mathrm{IC}_{50}$ in both cell lines, and the flow cytometric analyses showed an increase in the percentage of apoptotic cells as the concentration increased, also suggesting that apoptosis is one of the mechanisms by which cell death occurs after exposure to masitinib. Previous data suggest that the masitinib induces growth inhibition via apoptosis in various neoplastic canine cells $(6,15)$. These studies showed that masitinib at concentrations greater than $5 \mu \mathrm{M}$ and $10 \mu \mathrm{M}$ induces apoptosis in canine osteosarcoma cells and haemangiosarcoma cells respectively, whereas these concentrations are similar to our $\mathrm{IC}_{50}$ values.

Although the pathophysiology of TKIs causing apoptosis has not been clearly defined, other mechanisms in the apoptotic pathway such as DNA fragmentation may contribute to CMT cell death alongside masitinib. To elucidate the apoptotic pathway induced by masitinib in CMT cells, DNA fragmentation was analysed by the cytoplasmic histone-associated cell death method using ELISA. The results of the present study have proven that DNA fragmentation could be responsible for masitinibinduced apoptosis. It is possible that TKIs may downregulate the anti-apoptotic factors or upregulate the apoptotic factors or may directly process downstream signalling proteins in the apoptotic pathway.

The cell cycle distribution profiles of masitinib-treated CMT cells were examined over $72 \mathrm{~h}$ to investigate the mechanism underlying masitinib-mediated antiproliferative effects. The histograms of cell cycle analysis allow us to evaluate any change in the distribution of cell cycle phases by masitinib. Tyrosine kinase inhibitors appear to be more potent in arresting cancer cells in the $G_{0} / G_{1}$ phase, as reported previously for many other cancer cell lines $(29,30)$. Similarly to study findings previously reported, there was cell cycle arrest in $\mathrm{G}_{0} / \mathrm{G}_{1}$ with an associated decrease in the number of cells in the $\mathrm{S}$ phase, with the highest percentage of $\mathrm{G}_{0} / \mathrm{G}_{1}$ cells resulting when masitinib was used at nearly its $\mathrm{IC}_{50}$. The results 
of our study show that masitinib induces apoptosis and blocks cell-cycle progression in CMT cells. Both mechanisms may contribute to drug-induced growth inhibition in these cells. Additionally, it is plausible that alterations in cell cycle regulatory proteins such as downregulation of cyclin D1 may lead to the arrest of masitinib-treated CMT cells in the $\mathrm{G}_{0} / \mathrm{G}_{1}$ phase. Further investigation is needed to determine the expression of the relevant cell cycle regulatory proteins in masitinibtreated CMT cells.

In relation to the cell cycle results, the $\mathrm{Ki}-67$ marker allowing the estimation of the proportion of proliferative cells was detected (24). It has been reported that Ki-67 is the most frequently used prognostic biomarker in studies concerning canine mammary carcinomas and that its expression is the highest in tumours with poor clinical and histopathological characteristics $(10,19)$. It appears in the active phases of the cell cycle $\left(\mathrm{G}_{1}, \mathrm{~S}, \mathrm{G}_{2}\right.$, and $M$ ), but it is not expressed in the quiescent $\mathrm{G}_{0}$ phase (24). In the present study, consistently with our cell cycle analyses, less Ki-67 was also revealed to be in masitinibtreated cells due to the induction of cell arrest in $\mathrm{G}_{0} / \mathrm{G}_{1}$ phase. An explanation could be that the Ki-67 protein is absent in non-dividing masitinib-treated cells; the fact that cells which are not actively dividing cannot express Ki-67 has already been described in many cells (24). Battistello et al. (1) recently published results to which ours are similar; their observed outcome was masitinib reducing the proliferation of diffuse large B-cell lymphoma cells as evidenced by Ki-67 staining. Studies with different tyrosine kinase inhibitors on various cancer cells have shown accordant findings to the present study, demonstrating a decreasing proliferation index as measured by immunohistochemical staining for Ki-67 $(23,25)$.

Angiogenesis inhibition is an attractive anticancer strategy in cancer chemoprevention and therapy as it targets the vessels that provide oxygen and nutrients to actively proliferating cells. Vascular endothelial growth factor is a basic prerequisite for sustainable growth and proliferation of tumours and supports angiogenesis and proliferation and inhibition of apoptosis mediated by autocrine or paracrine loops between it and its receptors (17). Previous studies have demonstrated that dogs with mammary cancer have higher plasma VEGF concentrations than healthy dogs (22). In canine mammary tumours, overexpression of VEGF has been correlated with metastasis, and its role as a marker of malignancy and as a prognostic indicator has already been documented in canine mammary tumours $(11,22)$. It has been demonstrated in experimental studies that the inhibition of VEGF production by administration of TKIs was associated with decreased cell proliferation $(4,27)$. In the present study, VEGF was secreted heavily by CMT cells, predominantly by CMT-U27 cells, and treatment with masitinib contributed to a dosedependent inhibition of VEGF production by CMT-U27 and CMT-U309 cells. The difference between the VEGF levels of the two cell lines could be related to the doubling times of the CMT-U27 and CMT-U309 cells. The population-doubling time of the CMT-U27 cells is $48 \mathrm{~h}$; however, this value for CMT-U309 is $103.8 \mathrm{~h}$. Since the treatment period was $72 \mathrm{~h}$, the doubling time had been exceeded for CMT-U27 cells; however, the CMT-U309 cells had not yet completed this proliferation period. Additionally, the decrease in the levels of VEGF in the masitinib-treated cell lines may be related to a reduction in the density of the CMT cells. In contrast to our findings, Fahey et al. (6) reported that in vitro VEGF production by canine osteosarcoma cells was increased at around $\mathrm{IC}_{50}$ of masitinib in treated cells. In addition, elevated VEGF levels in cell supernatants close to the $\mathrm{IC}_{50}$ for masitinib were reported for canine haemangiosarcoma cell lines (15). These findings suggest that the higher VEGF level could have resulted from the cell membrane permeability that occurs with apoptosis. However, while in our study the reduced VEGF levels might partly be a consequence of the inhibition of proliferation of cancer cells by masitinib, they are more likely due to the decreased signalling in viable cells because in our study, the reduction in VEGF levels was not directly correlated with the decreased number of CMT cells. Although masitinib does not target the VEGF receptor, it is unknown whether it has the potential to regulate VEGF production from CMT cells by different mechanisms, hence there are several interdependent pathways driving angiogenesis in CMT cells. Finally, apart from KIT-dependent signalling pathways, other pro-oncogenic pathways may be associated with the effect of masitinib on canine mammary tumour cells.

In conclusion, masitinib inhibited the proliferation of CMT cells through induction of apoptosis and cell cycle arrest. Decreased immunocytochemical staining of masitinib-treated cells with $\mathrm{Ki}-67$ confirmed the antiproliferative effect of masitinib, in addition the reduced VEGF levels prove the ability of masitinib to modulate angiogenesis. Although the data obtained from this in vitro study need to be confirmed under in vivo conditions, we suggest that masitinib alone or in combination with cytotoxic chemotherapy will provide additional benefit in the treatment of canine mammary cancer.

Conflict of Interests Statement: The authors declare that there is no conflict of interests regarding the publication of this article.

Financial Disclosure Statement: This study was supported by the Research Fund of Istanbul University (Project number 7162).

Animal Rights Statement: None required.

Acknowledgement: We would like to thank $\mathrm{AB}$ Science for providing masitinib and we also thank Prof. Eva Hellmén for providing CMT cell lines. The authors would like to thank Colin D. Mansfield for his critical review of the present manuscript. A part of the present study was presented at the World Veterinary Cancer Congress 2016. 


\section{References}

1. Battistello E., Katanayeva N., Dheilly E., Tavernari D., Donaldson M.C., Bonsignore, L., Thome M., Christie A.L., Murakami M.A., Michielin O., Ciriello, G., Zoete V., Oricchio E.: Pan-SRC kinase inhibition blocks B-cell receptor oncogenic signaling in non-Hodgkin lymphoma. Blood 2018, 131, 23452356, doi: 10.1182/blood-2017-10-809210.

2. Bavcar S., Argyle D.J.: Receptor tyrosine kinase inhibitors: molecularly targeted drugs for veterinary cancer therapy. Vet Comp Oncol 2012, 10, 163-173, doi: 10.1111/j.14765829.2012.00342.x.

3. Chang C.Y., Chiou P.P., Chen W.J., Li Y.H., Yiu J.C., Cheng Y.H., Chen S.D., Lin C.T., Lai Y.S.: Assessment of the tumorigenesis and drug susceptibility of three new canine mammary tumor cell lines. Res Vet Sci 2010, 88, 285-293, doi: 10.1016/j.rvsc.2009. 08.006 .

4. Ciardiello F., Tortora G.: A novel approach in the treatment of cancer: targeting the epidermal growth factor receptor. Clin Cancer Res 2001, 7, 2958-2970.

5. Dubreuil P., Letard S., Ciufolini M., Gros L., Humbert M., Castéran N., Borge L., Hajem B., Lermet A., Sippl W., Voisset E., Arock M., Auclair C., Leventhal P.S., Mansfield C.D., Moussy A., Hermine O.: Masitinib (AB1010), a potent and selective tyrosine kinase inhibitor targeting KIT. PloS One 2009, 4, e7258, doi: 10.1371/journal.pone.0007258

6. Fahey C.E., Milner R.J., Kow K., Bacon N.J., Salute M.E.: Apoptotic effects of the tyrosine kinase inhibitor, masitinib mesylate, on canine osteosarcoma cells. Anti-Cancer Drugs 2013, 24, 519-526, doi: 10.1097/CAD.0b013e32836002ba.

7. Hahn K.A., Oglivie G., Rusk T., Devauchelle P., Leblanc A., Legendre A., Powers B., Leventhal P.S., Kinet J.P., Palmerini F., Dubreuil P., Moussy A., Hermine O.: Masitinib is safe and effective for the treatment of canine mast cell tumors. J Vet Intern Med 2008, 22, 1301-1309, doi: 10.1111/j.19391676.2008.0190.x.

8. Holtermann N., Kiupel M., Kessler M., Teske E., Betz D., Hirschberger J.: Masitinib monotherapy in canine epitheliotropic lymphoma. Vet Comp Oncol 2016, 14, 127-135, doi: 10.1111/ vco.12157.

9. Karayannopoulou M., Lafioniatis S.: Recent advances on canine mammary cancer chemotherapy: A review of studies from 2000 to date. Revue Méd Vét 2016, 167, 192-200.

10. Kaszak I., Ruszczak A., Kanafa S., Kacprzak K., Król M., Jurka P.: Current biomarkers of canine mammary tumors. Acta Vet Scand 2018, 60, 66, doi: 10.1186/s13028-018-0417-1.

11. Kato Y., Asano K., Mogi T., Kutara K., Teshima K., Edamura K., Tsumagari S., Hasegawa A., Tanaka S.: Clinical significance of circulating vascular endothelial growth factor in dogs with mammary gland tumors. J Vet Med Sci 2007, 69, 77-80, doi: 10.1292/jvms.69.77.

12. Koltai Z., Szabó B., Jakus J., Vajdovich P.: Tyrosine kinase expression analyses in canine mammary gland tumours - A pilot study. Acta Vet Hung 2018, 66, 294-308, doi: 10.1556/004.2018.027.

13. Kubo K., Matsuyama S., Katayama K., Tsutsumi C., Yonezawa K., Shimada T., Kotani T., Sakuma S., Ohashi F., Takamori Y.: Frequent expression of the c-kit proto-oncogene in canine malignant mammary tumor. J Vet Med Sci 1998, 60, 1335-1340, doi: $10.1292 /$ jvms.60.1335.

14. Lawrence J., Saba C., Gogal Jr R., Lamberth O., Vandenplas M.L., Hurley D.J., Dubreuil P., Hermine O., Dobbin K., Turek M.: Masitinib demonstrates anti-proliferative and pro-apoptotic activity in primary and metastatic feline injection-site sarcoma cells. Vet Comp Oncol 2012, 10, 143-154, doi: 10.1111/j.14765829.2011.00291.x.

15. Lyles S.E., Milner R.J., Kow K., Salute M.E.: In vitro effects of the tyrosine kinase inhibitor, masitinib mesylate, on canine hemangiosarcoma cell lines. Vet Comp Oncol 2012, 10, 223-235, doi: 10.1111/j.1476-5829.2012.00335.x.

16. Marech I., Patruno R., Zizzo N., Gadaleta C., Introna M., Zito A.F., Gadaleta C.D., Ranieri G.: Masitinib (AB1010), from canine tumor model to human clinical development: where we are? Crit Rev Oncol Hematol 2014, 91, 98-111, doi: 10.1016/j.critrevonc.2013.12.011.

17. Michishita M., Ohtsuka A., Nakahira R., Tajima T., Nakagawa T., Sasaki N., Arai T., Takahashi K.: Anti-tumor effect of bevacizumab on a xenograft model of feline mammary carcinoma. J Vet Med Sci 2016, 78, 685-694, doi: 10.1292/jvms.15-0550.

18. Milovancev M., Helfand S.C., Marley K., Goodall C.P., Löhr C.V., Bracha S.: Antiproliferative effects of masitinib and imatinib against canine oral fibrosarcoma in vitro. BMC Vet Res 2016, 12, 85, doi: 10.1186/s12917-016-0712-x.

19. Pena L.L., Nieto A.I., Pérez-Alenza D., Cuesta P., Castano M.: Immunohistochemical detection of Ki-67 and PCNA in canine mammary tumors: relationship to clinical and pathologic variables. J Vet Diagn Invest 1998, 10, 237-246, doi: $10.1177 / 104063879801000303$

20. Queiroga F.L., Raposo T., Carvalho M.I., Prada J., Pires I.: Canine mammary tumours as a model to study human breast cancer: most recent findings. In Vivo 2011, 25, 455-465.

21. Ranieri G., Gadaleta C.D., Patruno R., Zizzo N., Daidone M.G., Hansson M.G., Paradiso A., Ribatti D.: A model of study for human cancer: Spontaneous occurring tumors in dogs. Biological features and translation for new anticancer therapies. Crit Rev Oncol Hematol 2013, 88, 187-197, doi: 10.1016/j.critrevonc. 2013.03.005

22. Restucci B., Papparella S., Maiolino P., De Vico G.: Expression of vascular endothelial growth factor in canine mammary tumors. Vet Pathol 2002, 39, 488-493, doi: 10.1354/vp.39-4-488.

23. Sandström M., Johansson M., Andersson U., Bergh A., Bergenheim A.T., Henriksson R.: The tyrosine kinase inhibitor ZD6474 inhibits tumour growth in an intracerebral rat glioma model. Br J Cancer 2004, 91, 1174-1180, doi: 10.1038/sj.bjc. 6602108 .

24. Scholzen T., Gerdes J.: The Ki-67 protein: from the known and the unknown. J Cell Physiol 2000, 182, 311-322, doi: 10.1002/ (SICI)1097-4652(200003)182:3<311::AID-JCP1>3.0.CO;2-9.

25. Stabile L.P., Lyker J.S., Gubish C.T., Zhang W., Grandis J.R., Siegfried J.M.: Combined targeting of the estrogen receptor and the epidermal growth factor receptor in non-small cell lung cancer shows enhanced antiproliferative effects. Cancer Res 2005, 65, 1459-1470, doi: 10.1158/0008-5472.CAN-04-1872.

26. Thamm D.H., Rose B., Kow K., Humbert M., Mansfield C.D., Moussy A., Hermine O., Dubreuil P.: Masitinib as a chemosensitizer of canine tumor cell lines: a proof of concept study. Vet J 2012, 191, 131-134, doi: 10.1016/j.tvj1.2011.01.001.

27. Wilhelm S.M., Adnane L., Newell P., Villanueva A., Llovet J.M., Lynch M.: Preclinical overview of sorafenib, a multikinase inhibitor that targets both Raf and VEGF and PDGF receptor tyrosine kinase signaling. Mol Cancer Ther 2008, 7, 3129-3140, doi: 10.1158/1535-7163.MCT-08-0013.

28. Wolfesberger B., Tonar Z., Gerner W., Skalicky M., Heiduschka G., Egerbacher M., Thalhammer J.G., Walter I.: The tyrosine kinase inhibitor sorafenib decreases cell number and induces apoptosis in a canine osteosarcoma cell line. Res Vet Sci 2010, 88, 94-100, doi: 10.1016/j.rvsc.2009.06.009.

29. Xiao X., Wu J., Zhu X., Zhao P., Zhou J., Liu Q.Q., Zheng L., Zeng M., Liu R., Huang W.: Induction of cell cycle arrest and apoptosis in human nasopharyngeal carcinoma cells by ZD6474, an inhibitor of VEGFR tyrosine kinase with additional activity against EGFR tyrosine kinase. Int J Cancer 2007, 121, 2095-2104, doi: 10.1002/ijc.22955.

30. Zhou X., Zheng M., Chen F., Zhu Y., Yong W., Lin H., Sun Y., Han X.: Gefitinib inhibits the proliferation of pancreatic cancer cells via cell cycle arrest. Anat Rec (Hoboken) 2009, 292, 1122-1127, doi: 10.1002/ar.20938. 